\title{
Health status of middle-aged and older cancer survivors: a nationwide cross-sectional study from the China Health and Retirement Longitudinal Study (CHARLS)
}

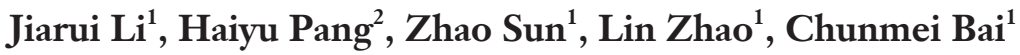 \\ ${ }^{1}$ Department of Medical Oncology, Peking Union Medical College Hospital, Chinese Academy of Medical Sciences and Peking Union Medical \\ College, Beijing 100032, China; ${ }^{2}$ Central Research Laboratory, Peking Union Medical College Hospital, Chinese Academy of Medical Sciences and \\ Peking Union Medical College, Beijing 100730, China \\ Contributions: (I) Conception and design: J Li, L Zhao, C Bai; (II) Administrative support: J Li, L Zhao, C Bai; (III) Provision of study materials or \\ patients: J Li; (IV) Collection and assembly of data: J Li; (V) Data analysis and interpretation: J Li, H Pang; (VI) Manuscript writing: All authors; (VII) \\ Final approval of manuscript: All authors. \\ Correspondence to: Lin Zhao; Chunmei Bai. Department of Medical Oncology, Peking Union Medical College Hospital, Chinese Academy of Medical \\ Sciences and Peking Union Medical College, Beijing 100032, China. Email: wz20010727@aliyun.com; baichunmei1964@163.com.
}

\begin{abstract}
Background: The number of Chinese cancer survivors has increased bolstered by the combined trends of an aging population and improved cancer survival; however, related research on cancer survivorship remains limited. Therefore, this study aimed to provide an overview of the health status of middle-aged and older cancer survivors in China.

Methods: We used the cross-sectional self-reported survey data from wave 4 in the China Health and Retirement Longitudinal Study (CHARLS). A total of 354 cancer survivors and 16,664 participants without cancer were identified from CHARLS. Physical and mental health, health behavior, and health care information collected by questionnaire were compared between these two groups.

Results: Compared with the general middle-aged and older population, cancer survivors had more concomitant chronic diseases $(2.75$ vs. 2.00, $\mathrm{P}<0.001)$. In addition, cancer survivors were found to be more statistically likely to have difficulties with activity $(3.53$ vs. $2.39, \mathrm{P}<0.001)$ and have depressive symptoms $(10.07$ vs. 8.01, $\mathrm{P}<0.001)$ compared with participants without cancer. Also, cancer survivors were less likely to drink compared to those without a cancer diagnosis (OR 0.49; 95\% CI, 0.36-0.66, $\mathrm{P}<0.001$ ), but smoking behavior and physical activity did not show a significant difference. Coexisting chronic diseases and smoking harmed the physical and mental health of middle-aged and older people. We also found that cancer survivors had higher medical care expenses when compared with participants without cancer.

Conclusions: Cancer survivors older than 45 years in China have poorer outcomes in comorbidities and physical and mental health than their age-matched individual counterparts without cancer. Therefore, a higher quality and more cost-effective supportive care for these individuals is needed.
\end{abstract}

Keywords: Cancer survivor; health status; quality of life; China Health and Retirement Longitudinal Study (CHARLS)

Submitted Oct 28, 2019. Accepted for publication Jan 14, 2020.

doi: $10.21037 /$ atm.2020.01.105

View this article at: http://dx.doi.org/10.21037/atm.2020.01.105 


\section{Introduction}

In 2015, about 4.3 million Chinese people were diagnosed with cancer and the prevalence of this malignant disease was predicted to increase in the future due to population growth and aging (1). Meanwhile, the rate of cancer survival in China has increased remarkably in the past 10 years (2). In China, about $40.5 \%$ of cancer survivors can live for more than 5 years and some even view their cancer as a chronic disease. However, cancer survivors live with continuous severe physical and mental health problems, which impair their quality of life (3-7). In addition, with the increasing cost of medical insurance coverage, the economic burden of cancer is also a severe challenge for the medical and health care system in China $(8,9)$. Therefore, estimating the health status of Chinese cancer survivors and providing scientific evidence for future health care reform is urgently required. Previous studies have concentrated on the health-related quality of life among Chinese cancer survivors, but most studies have focused on a single cancer type and lacked age-matched comparison groups, making it difficult to determine whether it was the cancer diagnosis or senescence that was responsible for the poor condition of the patients (10-15).

Therefore, this study was designed to provide an overview of the health status of middle-aged and older cancer survivors from a prospective population-based study in China. We will investigate the physical and mental health, health behavior, and health care information of cancer survivors older than 45 years.

\section{Methods}

\section{Study population}

Data for analysis were collected from the Harmonized China Health and Retirement Longitudinal Study (CHARLS). The CHARLS, run by the China Center for Economic Research at Peking University, contains the national records of individuals older than 45 years in China and aims to provide a high-quality national database of Chinese residents. The study serves the needs of scientific research for age-related issues. The detail of the sampling procedure and descriptions from the CHARLS are described elsewhere (16). The baseline wave of the CHARLS was conducted in 2011-2012, and the individuals involved were followed up every 2 years. This initial sample covered 10,257 households in 150 counties/ districts and 450 villages or urban communities across 28 provinces. All participants had a face-to-face interview using a structured questionnaire, which included assessments on the participants social, economic, and health status, among other issues. The latest survey (Wave 4) data in 2015 was adopted for the current cross-sectional study, and the response rate was $82.7 \%$. A total of 17,018 individuals were analyzed in this study after 4,079 participants were excluded because data were missing from the cancer history report.

The CHARLS was approved by the ethics committee of Peking University Health Science Center. Written informed consent was obtained from each participant.

\section{Measurements and definitions}

\section{Demographic characteristics and health status}

Participants reported their age, gender, marital status, living region, and education. Participants were asked if a doctor had ever informed them that they had cancer or a malignant tumor. Participants who answered "yes" were defined as cancer survivors. The participants were also asked if they had been diagnosed with any other chronic diseases. In this study, we considered hypertension, diabetes or high blood sugar, chronic lung disease (chronic bronchitis or emphysema), heart problems (heart attack, coronary heart disease, angina, and congestive heart failure), stroke, psychiatric problems, arthritis, dyslipidemia (elevation of low-density lipoprotein, triglycerides, and total cholesterol, or a low high-density lipoprotein level), liver disease (except fatty liver and tumor), kidney disease (except for tumor), stomach or other digestive disease (except for tumor), and asthma as chronic diseases.

Participants were asked about their current health condition by using a scale, ranging from 1 for excellent (very good) to 5 for poor (very bad). Daily activity was evaluated by using health variables combined from the Harmonized CHARLS database, and included activities of daily life, instrumental activities of daily life, and mobility summaries. A higher daily activity score suggested that the participants had more difficulties in physical activities in their daily life. Cognitive function was assessed by the Telephone Interview of Cognitive Status (TICS) test and by examining the figure drawings in CHARLS, with a higher cognitive function score representing a better performance by a participant in the cognition function test (17). Depression was assessed using the Center for Epidemiologic Studies Depression Scale (CES-D) with 10 items (18). The CES-D-10 ranges from 0 to 30 with a higher score indicating that the respondent has experienced more depressive symptoms in 
the previous week.

\section{Health behavior}

Participants who had ever smoked in the past were defined as former smokers, and participants who were still smoking were defined as current smokers. Similarly, participants who reported they had an alcoholic drink in the past were defined as former drinkers, and participants who consumed any alcohol in the last year were defined as current drinkers. Physical activity status was categorized as taking part in vigorous or moderate activity more than once a week.

\section{Health care use and costs}

Health care information from CHARLS was obtained through interviewing participants. All participants were asked whether they received inpatient care in the past year or outpatient care in the past month. If the participant received inpatient or outpatient care, they were asked to provide information on the value of the out-of-pocket and total medical cost.

\section{Statistical analysis}

The statistical analyses were performed with Stata (version 15.1, Stata Corp., College Station, TX, USA) and SPSS (version 25, IBM Corp., Armonk, NY, USA). Characteristics of cancer survivors and participants without cancer were compared using Student's $t$-test for normally distributed continuous variables and chi-squared test or Mann-Whitney rank-sum test for categorical variables. The results of self-reported health, daily activity, cognitive function, and depression were also presented, and adjusted for age, sex, living region, and concomitant diseases using multivariate logistic regression analysis. We used binary logistic regression to calculate the odds ratio (OR) for health behavior in cancer survivors versus those with no cancer history and then adjusted for variables that might affect health behavior. We employed a multiple linear regression model to examine the influence of related demographic characteristics (age, sex and living region), coexisting chronic conditions, and health behavior (smoking and drinking) on daily activity, cognitive function and depression scores of cancer survivors and participants without cancer. A P value less than 0.05 (two-sided test) was considered to be statistically significant.

\section{Results}

\section{Demographic characteristics and bealth status}

There were 354 cancer survivors in this analysis (2.1\%) and 16,664 participants who did not report a history of cancer. The five most diagnosed cancers were breast $(18.5 \%)$, endometrium $(14.8 \%)$, colorectum (11.0\%), cervix $(9.5 \%)$, and esophagus (9.0\%), accounting for more than $60 \%$ of all reported cancer cases. In the past two years, more than half of these survivors $(55.7 \%)$ reported that they took Western modern medicine to relieve related symptoms, and a third of the survivors took Chinese traditional medicine. About $48.1 \%$ of cancer survivors received surgery, $28.6 \%$ of them received chemotherapy, and $31.0 \%$ of them reported they did not receive any treatment in the past 2 years.

The demographic characteristics of cancer survivors and participants without cancer are shown in Table 1. The mean age of cancer survivors was 61.0 years. A higher percentage of cancer survivors were female $(68.9 \%$ vs. $52.3 \%, \mathrm{P}<0.001)$ and lived in a rural region $(43.8 \%$ vs. $38.4 \%, \mathrm{P}=0.039)$ than those without cancer. Cancer survivors also had more concomitant chronic diseases than participants without cancer (2.75 vs. 2.00, $\mathrm{P}<0.001)$. Cancer survivors and participants without cancer did not show significant differences in age, marital status, education, or body mass index.

The current health condition reported by cancer survivors themselves was poorer than that reported by participants without cancer $(\mathrm{P}<0.001)$. The daily activity score was higher in the cancer group $(\mathrm{P}<0.001)$, which indicated that cancer survivors had more difficulties in their daily life than other middle-aged and older participants. There was no statistically significant difference in cognition between these two groups even after adjustment $(\mathrm{P}=0.612)$. Cancer survivors had a significantly higher CES-D score after adjustment, which means they had more depressive symptoms than participants without cancer $(\mathrm{P}=0.003)$.

The coexisting diseases of cancer survivors and other middle-aged and older participants are shown in Figure 1. More than $45 \%$ of cancer survivors had 3 or more chronic diseases. Cancer survivors more frequently reported having arthritis $(51.8 \%$ vs. $45.4 \%, \mathrm{P}=0.020)$, stomach or digestive disease $(43.1 \%$ vs. $32.6 \%, \mathrm{P}<0.001)$, cardiovascular disorders (29.6\% vs. 18.7\%, $\mathrm{P}<0.001)$, dyslipidemia (27.0\% vs. $18.2 \%$, $\mathrm{P}<0.001)$, diabetes or high blood sugar $(21.0 \%$ vs. $10.2 \%$, 
Table 1 Baseline characteristics of middle-aged and older cancer survivors and participants without cancer from CHARLS

\begin{tabular}{|c|c|c|c|c|c|}
\hline Characteristic & $\begin{array}{c}\text { Total } \\
(n=17,018)\end{array}$ & $\begin{array}{l}\text { Cancer survivors } \\
\qquad(\mathrm{n}=354)\end{array}$ & $\begin{array}{l}\text { No cancer } \\
(n=16,664)\end{array}$ & $P$ value & $P$ value $^{\dagger}$ \\
\hline Age (years), mean $\pm S D$ & $61.02 \pm 10.06$ & $60.97 \pm 10.35$ & $61.02 \pm 10.05$ & 0.355 & \\
\hline Male & $8,062(47.4)$ & $110(31.1)$ & $7,952(47.7)$ & & \\
\hline Female & $8,956(52.6)$ & $224(68.9)$ & $8,712(52.3)$ & & \\
\hline Urban & $10,464(61.5)$ & 199 (56.2) & $10,265(61.6)$ & & \\
\hline Married, n (\%) & $13,619(80.0)$ & 295 (83.3) & $13,324(80.0)$ & 0.116 & \\
\hline Education, n (\%) & & & & 0.181 & \\
\hline$\leq$ Primary school & $11,309(66.5)$ & $247(69.8)$ & $11,062(66.4)$ & & \\
\hline Self-reported health score [1-5], mean \pm SD & $3.39 \pm 0.38$ & $3.87 \pm 0.82$ & $3.38 \pm 0.87$ & $<0.001$ & $<0.001$ \\
\hline Daily activity, mean \pm SD & $2.41 \pm 3.36$ & $3.53 \pm 3.85$ & $2.39 \pm 3.35$ & $<0.001$ & $<0.001$ \\
\hline Cognitive function, mean \pm SD & $12.89 \pm 5.69$ & $12.61 \pm 5.60$ & $12.90 \pm 5.69$ & 0.629 & 0.612 \\
\hline Depression, mean \pm SD & $8.05 \pm 6.41$ & $10.07 \pm 7.06$ & $8.01 \pm 6.39$ & $<0.001$ & 0.003 \\
\hline
\end{tabular}

${ }^{\dagger}$, adjusted for age, sex, region, and number of concomitant chronic diseases. CHARLS, China Health and Retirement Longitudinal Study.

A
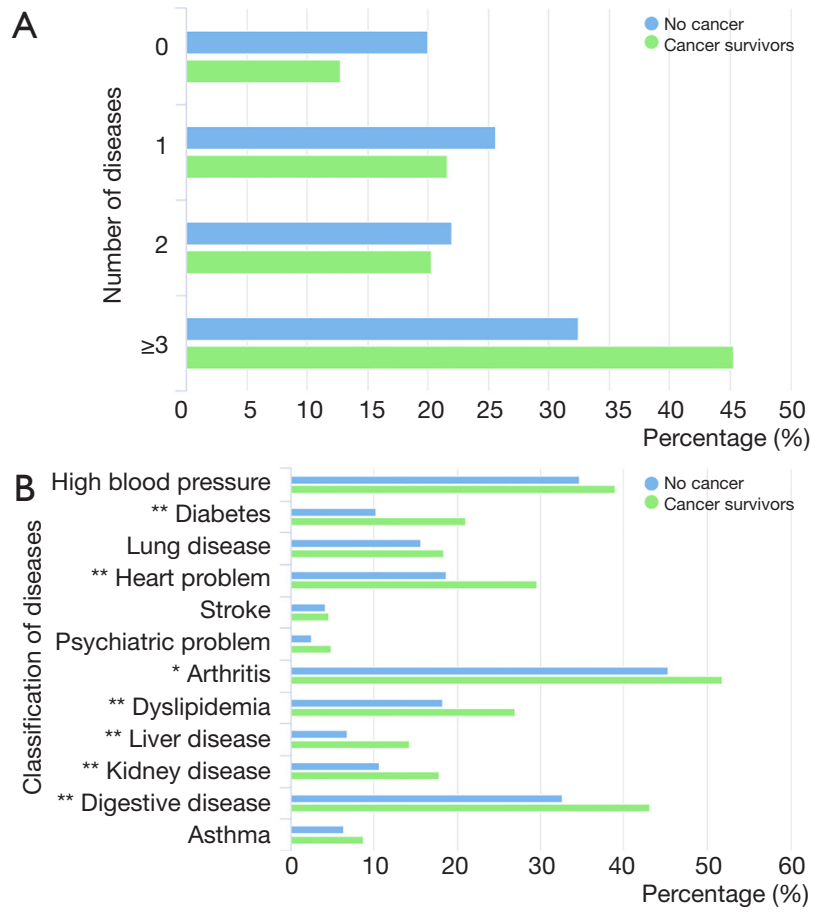

Figure 1 Concomitant chronic diseases of cancer survivors and participants without cancer. (A) Number of chronic diseases (except for cancer); (B) prevalence of different types of chronic diseases. *, $\mathrm{P}<0.05 ;{ }^{* *}, \mathrm{P}<0.001$.
$\mathrm{P}<0.001)$, renal diseases $(17.9 \%$ vs. $10.6 \%, \mathrm{P}<0.001)$, and liver diseases $(14.2 \%$ vs. $6.8 \%, \mathrm{P}<0.001)$ than participants without cancer. The prevalence of other chronic diseases was similar between the two groups.

\section{Health behavior}

The health behaviors of the participants are presented in Table 2. After adjusting for age, sex, living region and chronic diseases, there were no significant differences found among former (OR 0.97; 95\% CI, 0.67-1.39, $\mathrm{P}=0.853$ ) and current smoking behavior (OR 0.75; 95\% CI, 0.52-1.08, $\mathrm{P}=0.121$ ) between cancer survivors and participants without cancer. The proportion of cancer survivors who reported having an alcoholic beverage in the last 12 months was lower compared with those with no history of cancer $(17.3 \%$ vs. $34.7 \%, \mathrm{P}<0.001)$, and the difference was significant after adjusting for age, sex, living region and chronic diseases (OR 0.49; 95\% CI, 0.36-0.66, $\mathrm{P}<0.001$ ). In addition, 58.6\% of cancer survivors engaged in vigorous or moderately energetic physical activity for at least 10 minutes every week compared with $65.4 \%$ of those without a cancer diagnosis, but this difference was not significant. 
Table 2 Health behaviors of middle-aged and older cancer survivors and participants without cancer from CHARLS

\begin{tabular}{|c|c|c|c|}
\hline Characteristic & n (\%) & OR (95\% Cl) & OR $(95 \% \mathrm{Cl})^{\dagger}$ \\
\hline No cancer & $7,380(44.3)$ & 1.00 & 1.00 \\
\hline Cancer survivors & $115(32.7)$ & $0.61(0.49-0.76)^{*}$ & $0.97(0.67-1.39)$ \\
\hline \multicolumn{4}{|l|}{ Current smoker } \\
\hline Cancer survivors & $58(16.5)$ & $0.51(0.38-0.68)^{*}$ & $0.75(0.52-1.08)$ \\
\hline \multicolumn{4}{|l|}{ Former drinker } \\
\hline No cancer & $7,671(46.1)$ & 1.00 & 1.00 \\
\hline Cancer survivors & $127(36.1)$ & $0.66(0.53-0.82)^{\star}$ & $0.88(0.67-1.16)$ \\
\hline Cancer survivors & $61(17.3)$ & $0.39(0.30-0.52)^{*}$ & $0.51(0.37-0.72)$ * \\
\hline \multicolumn{4}{|l|}{ Physical activity } \\
\hline No cancer & $5,142(65.4)$ & 1.00 & 1.00 \\
\hline Cancer survivors & 99 (58.6) & $0.75(0.55-1.02)$ & $0.75(0.53-1.06)$ \\
\hline
\end{tabular}

${ }^{\dagger}$, adjusted for age, sex, region and number of concomitant chronic diseases; ${ }^{*}, \mathrm{P}<0.001$.

\section{Daily activity, cognitive function and depression}

The multiple linear regression results of daily activity, cognitive function, and depression are shown in Table 3. The number of concomitant chronic diseases was associated with more physical limitations in daily activities and depressive symptoms in both the cancer and without cancer groups (all $\mathrm{P}<0.001)$. However, such an association was not found in cognitive function. Current smoking behavior had a negative impact on cognitive function and depression in both the cancer and without cancer groups, but the influence of smoking was quite limited in the without cancer group. In participants without cancer, drinking alcohol had a minor protective effect on daily activity, cognitive function, and depression.

\section{Health care use and costs}

Table 4 provides data on medical care utilization and expenditure. Cancer survivors received inpatient or outpatient care more frequently than participants without cancer $(\mathrm{P}<0.001)$. In addition, cancer survivors had significantly higher out-of-pocket or total medical costs of the hospitalization or doctor visit compared to those without cancer $(\mathrm{P}<0.001)$. More than $90 \%$ of participants were covered by public health insurance, but only a minority of participants $(2.3 \%)$ had private health insurance.

\section{Discussion}

This study used prospective population-based samples of middle-aged and older participants to evaluate the general health status of cancer survivors in China. Cancer survivors reported poor physical and mental health status. They had more physical limitations in completing activities in their daily life and experienced more depressive symptoms than the general population; however, it was found that they did not have a higher prevalence of cognitive impairment. Cancer survivors also had higher medical expenditures than participants without cancer.

Previous studies have suggested that cancer survivors are physically active and are more prone to developing psychosocial problems and cognitive impairment than those without cancer; furthermore, their needs have not been addressed appropriately (19-25). Unfortunately, cancer survivors older than 45 years have a high prevalence of co-existing chronic diseases, and more than $45 \%$ of the present study's population had 3 or more comorbidities. A 


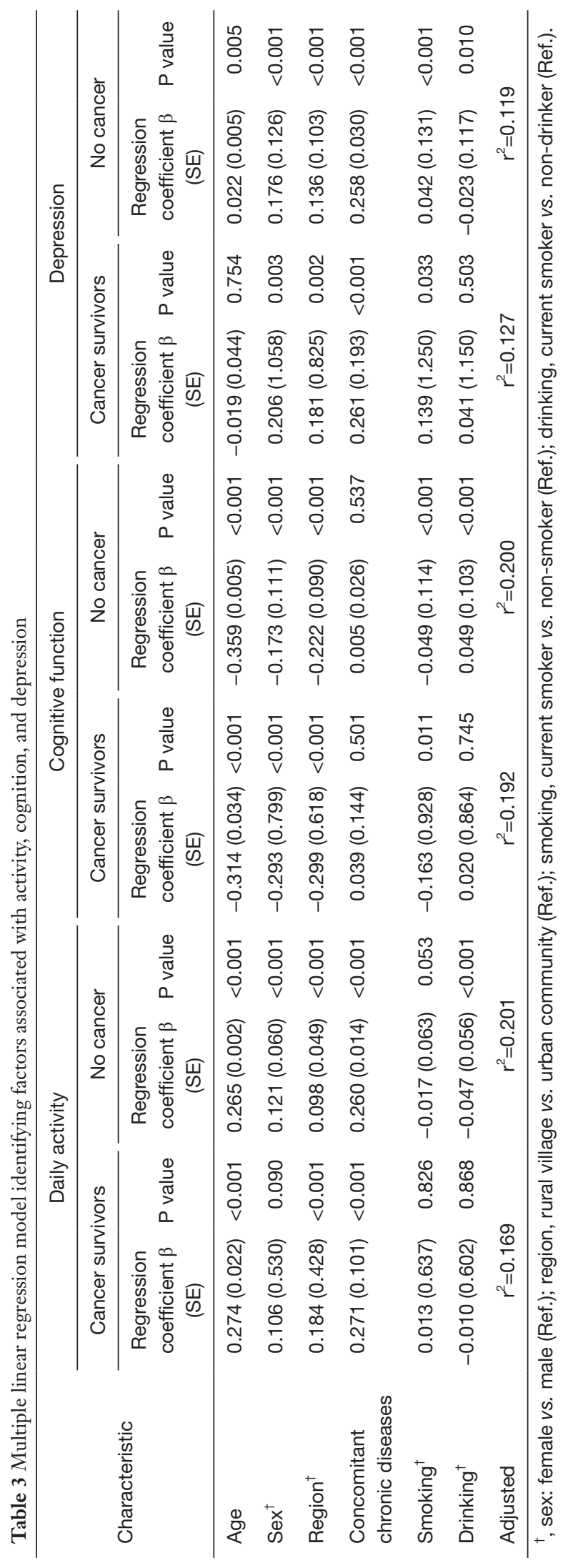

similar concomitant illness burden of cancer survivors has also been reported in other countries, but chronic diseases beyond cancer have just begun to receive attention in clinical research $(6,7,26,27)$. Several studies have indicated that chronic diseases, such as diabetes, could increase the risk of all-cause mortality in patients with cancer (28-30). The regression model from this present study showed that concomitant chronic diseases had a negative impact on daily activities, cognitive functions, and the mental health of the middle-aged and older population, which indicate that cancer survivors with chronic diseases have more difficulty performing self-care tasks and lacked the ability to live independently. Therefore, collaborative care is essential to ensuring medical conditions beyond cancer are well treated, and this collaboration should include not only oncologists but also supportive care providers and mental health specialists.

Health behaviors, such as smoking, drinking, and physical inactivity, have a large impact on health status not only in the middle-aged and older populations but also in cancer survivors (31-34). In addition, this research indicated that smoking behavior has an unfavorable impact on cognition function and mental health in both cancer and no-cancer groups, which has been reported in several prior studies (35-37). Our study found that there were no statistically significant differences in cigarette smoking between cancer survivors and those without cancer after adjustment. Also, cancer survivors who continue to smoke after a cancer diagnosis are at an increased risk for subsequent cancers and have a significantly higher symptom burden than nonsmokers $(31,38,39)$. Although the public recognition of the hazards of smoking has improved in the past 15 years in China, the government still needs to broaden the awareness and intensify the education concerning the effects of smoking on the physical and psychosocial outcomes (40).

Compared with participants without cancer, Chinese cancer survivors were less likely to be current drinkers, which was different from western cancer survivors (19,41-43). The linear regression model from the present study showed that drinking had a minor benefit on the physical and mental health of participants without cancer; however, such a benefit was not seen in cancer survivors, and previous studies have observed that alcohol intake increased cancerrelated mortality $(32,44)$. A recent nationwide study showed that smoking, infections, and poor diet were the largest contributors to the total cancer burden in China (45). Therefore, it is still imperative for clinicians to encourage cancer survivors to engage in a healthy lifestyle to improve 
Table 4 Health care utilization and insurance of the cohort from CHARLS

\begin{tabular}{|c|c|c|c|c|c|}
\hline Characteristic & Total & Cancer survivors & No cancer & $P$ value & $P$ value \\
\hline Doctor visit/outpatient last month, n (\%) & $3,193(18.8)$ & $110(32.3)$ & $3,083(19.1)$ & $<0.001$ & $<0.001$ \\
\hline $\begin{array}{l}\text { Hospitalization out-of-pocket expenditure } \\
\text { last year (yuan), mean } \pm S D\end{array}$ & $7,736.5 \pm 16,677.0$ & $17,547.2 \pm 26,958.6$ & $7,282.1 \pm 15,906.1$ & $<0.001$ & $<0.001$ \\
\hline $\begin{array}{l}\text { Doctor visit out-of-pocket expenditure last } \\
\text { month (yuan), mean } \pm S D\end{array}$ & $1,056.2 \pm 3,963.4$ & $3,047.6 \pm 9,190.9$ & $987.7 \pm 3,627.8$ & $<0.001$ & $<0.001$ \\
\hline $\begin{array}{l}\text { Doctor visit total expenditure last month } \\
\text { (yuan), mean } \pm S D\end{array}$ & $1,488.5 \pm 5,228.0$ & $3,952.4 \pm 10,521.2$ & $1,401.8 \pm 4,921.2$ & $<0.001$ & $<0.001$ \\
\hline
\end{tabular}

${ }^{\dagger}$, adjusted for age, sex, region, and number of concomitant chronic diseases.

their health status $(46,47)$.

Cancer inflicts a heavy health burden on middle-aged and older people. Surprisingly, we found about $70 \%$ of cancer survivors did not visit hospitals in their last year of followup. Although public health insurance was available to almost all middle-aged and older people and paid on average $40 \%$ of the total medical cost for insured patients, the inpatient and outpatient payments were still a substantial economic burden for patients who went to the hospital. The largest component of the total economic burden was the cost of hospitalization, and the out-of-pocket medical expense for hospitalization in the last year for those with cancer was $¥ 17,547$ compared with $¥ 7,282$ for those without cancer. According to a 2015 Chinese study, the total payments on cancer treatments were estimated to be 221.4 billion RMB per year, accounting for $17.7 \%$ of the government health expenditure (8). In our study, only $30 \%$ of cancer patients visited the hospital last year, and the total expenditure on cancer treatment would become much higher if these patients were insured. The weighty economic burden of cancer is not affordable to the current Chinese government, which indicates that China should maintain a high growth rate and deepen the reforms of the current health care system. We observed that private health insurance covers only a few middle-aged and older people. To further reduce the economic burden of health care, private insurance might provide extra financial protection to some wealthy people with the greatest need.

This study has several limitations. First, cancer diagnosis and other health status information were reported only by the participants themselves. Second, some important factors, such as the age of cancer diagnosis, tumor stage, and cancer-related treatment regimen, were not available in the CHARLS study, which might have an impact on the health status and health service utilization of cancer survivors. Third, health behaviors were also self-reported, and some participants might have concealed their real cigarette and alcohol consumption. Finally, the CHARLS included only 21,097 participants, which limited the generalization of this study.

Nonetheless, our study is a first step toward providing a comprehensive and up-to-date assessment of the general health status of middle-aged and older cancer survivors in China. The poor health status reported in our study highlights the need for collaboration between oncologists and supportive care providers (such as general practitioners) to find effective strategies to improve the physical and psychological well-being of the growing numbers of cancer survivors.

\section{Conclusions}

In conclusion, Chinese cancer survivors older than 45 years report poor general health status in physical and mental health. They also have higher medical expenses than noncancer participants. Because of the growing number of cancer survivors and their heavy illness burden, high-quality and cost-effective supportive care provided by the health 
care system is needed in the future.

\section{Acknowledgments}

This analysis used data or information from the Harmonized CHARLS dataset and Codebook, Version C, as of April 2018 developed by the Gateway to Global Aging Data. The development of the Harmonized CHARLS was funded by the National Institute on Ageing (R01 AG030153, RC2 AG036619, R03 AG043052). For more information, please refer to www.g2aging.org.

Funding: This work was supported by grants from the National Natural Science Foundation of China (No. 61435001) and CAMS Innovation Fund for Medical Sciences (No. 2016-I2M-1-001, No. 2017-I2M-4-003).

\section{Footnote}

Conflicts of Interest: The authors have no conflicts of interest to declare.

Ethical Statement: The authors are accountable for all aspects of the work in ensuring that questions related to the accuracy or integrity of any part of the work are appropriately investigated and resolved. The CHARLS was approved by the ethics committee of Peking University Health Science Center. Written informed consent was obtained from each participant.

Open Access Statement: This is an Open Access article distributed in accordance with the Creative Commons Attribution-NonCommercial-NoDerivs 4.0 International License (CC BY-NC-ND 4.0), which permits the noncommercial replication and distribution of the article with the strict proviso that no changes or edits are made and the original work is properly cited (including links to both the formal publication through the relevant DOI and the license). See: https://creativecommons.org/licenses/by-nc-nd/4.0/.

\section{References}

1. Chen $\mathrm{W}$, Zheng R, Baade PD, et al. Cancer statistics in China, 2015. CA Cancer J Clin 2016;66:115-32.

2. Zeng $\mathrm{H}$, Chen $\mathrm{W}$, Zheng R, et al. Changing cancer survival in China during 2003-15: a pooled analysis of 17 population-based cancer registries. Lancet Glob Health 2018;6:e555-67.
3. Mayer DK, Nasso SF, Earp JA. Defining cancer survivors, their needs, and perspectives on survivorship health care in the USA. Lancet Oncol 2017;18:e11-8.

4. Stein KD, Syrjala KL, Andrykowski MA. Physical and psychological long-term and late effects of cancer. Cancer 2008;112:2577-92.

5. Hewitt M, Greenfield S, Stovall E. From cancer patient to cancer survivor: lost in transition. Washington, DC: National Academies Press, 2005.

6. Sulicka J, Pac A, Puzianowska-Kuznicka M, et al. Health status of older cancer survivors-results of the PolSenior study. J Cancer Surviv 2018;12:326-33.

7. Holmes HM, Nguyen HT, Nayak P, et al. Chronic conditions and health status in older cancer survivors. Eur J Intern Med 2014;25:374-8.

8. Cai Y, Xue M, Chen W, et al. Expenditure of hospital care on cancer in China, from 2011 to 2015. Chin J Cancer Res 2017;29:253-62.

9. Meng Q, Xu L, Zhang Y, et al. Trends in access to health services and financial protection in China between 2003 and 2011: a cross-sectional study. Lancet 2012;379:805-14.

10. Su M, Hua X, Wang J, et al. Health-related quality of life among cancer survivors in rural China. Qual Life Res 2019;28:695-702.

11. Wang T, Jiang M, Ren Y, et al. Health-Related Quality of Life of Community Thyroid Cancer Survivors in Hangzhou, China. Thyroid 2018;28:1013-23.

12. Wang JW, Sun L, Li J, et al. Comorbid chronic diseases and their associations with quality of life among gynecological cancer survivors. BMC Public Health 2015; 15:965.

13. Yan YB, Meng L, Liu ZQ, et al. Quality of life in longterm oral cancer survivors: an 8-year prospective study in China. Oral Surg Oral Med Oral Pathol Oral Radiol 2017;123:67-75.

14. Gong XH, Wang JW, Li J, et al. Physical exercise, vegetable and fruit intake and health-related quality of life in Chinese breast cancer survivors: a cross-sectional study. Qual Life Res 2017;26:1541-50.

15. Xia J, Tang Z, Deng Q, et al. Predictors of the quality of life in Chinese breast cancer survivors. Breast Cancer Res Treat 2018;167:537-45.

16. Zhao Y, Hu Y, Smith JP, et al. Cohort profile: the China Health and Retirement Longitudinal Study (CHARLS). Int J Epidemiol 2014;43:61-8.

17. Knopman DS, Roberts RO, Geda YE, et al. Validation of the telephone interview for cognitive status-modified in 
subjects with normal cognition, mild cognitive impairment, or dementia. Neuroepidemiology 2010;34:34-42.

18. Andresen EM, Malmgren JA, Carter WB, et al. Screening for depression in well older adults: evaluation of a short form of the CES-D (Center for Epidemiologic Studies Depression Scale). Am J Prev Med 1994;10:77-84.

19. Grimmett C, Wardle J, Steptoe A. Health behaviours in older cancer survivors in the English Longitudinal Study of Ageing. Eur J Cancer 2009;45:2180-6.

20. Blanchard CM, Courneya KS, Stein K, et al. Cancer survivors' adherence to lifestyle behavior recommendations and associations with health-related quality of life: results from the American Cancer Society's SCS-II. J Clin Oncol 2008;26:2198-204.

21. Andersen BL, DeRubeis RJ, Berman BS, et al. Screening, assessment, and care of anxiety and depressive symptoms in adults with cancer: an American Society of Clinical Oncology guideline adaptation. J Clin Oncol 2014;32:1605-19.

22. Foster C, Wright D, Hill H, et al. Psychosocial implications of living 5 years or more following a cancer diagnosis: a systematic review of the research evidence. Eur J Cancer Care (Engl) 2009;18:223-47.

23. Stanton AL. What happens now? Psychosocial care for cancer survivors after medical treatment completion. J Clin Oncol 2012;30:1215-20.

24. Ahles TA, Root JC, Ryan EL. Cancer- and cancer treatment-associated cognitive change: an update on the state of the science. J Clin Oncol 2012;30:3675-86.

25. Li M, Kennedy EB, Byrne N, et al. Systematic review and meta-analysis of collaborative care interventions for depression in patients with cancer. Psychooncology 2017;26:573-87.

26. Yabroff KR, Lawrence WF, Clauser S, et al. Burden of illness in cancer survivors: findings from a population-based national sample. J Natl Cancer Inst 2004;96:1322-30.

27. Ness KK, Wall MM, Oakes JM, et al. Physical performance limitations and participation restrictions among cancer survivors: a population-based study. Ann Epidemiol 2006;16:197-205.

28. Barone BB, Yeh HC, Snyder CF, et al. Long-term allcause mortality in cancer patients with preexisting diabetes mellitus: a systematic review and meta-analysis. JAMA 2008;300:2754-64.

29. Wu AH, Kurian AW, Kwan ML, et al. Diabetes and other comorbidities in breast cancer survival by race/ ethnicity: the California Breast Cancer Survivorship Consortium (CBCSC). Cancer Epidemiol Biomarkers Prev 2015;24:361-8.

30. Yancik R, Wesley MN, Ries LA, et al. Effect of age and comorbidity in postmenopausal breast cancer patients aged 55 years and older. JAMA 2001;285:885-92.

31. Peppone LJ, Mustian KM, Morrow GR, et al. The effect of cigarette smoking on cancer treatment-related side effects. Oncologist 2011;16:1784-92.

32. Jin M, Cai S, Guo J, et al. Alcohol drinking and all cancer mortality: a meta-analysis. Ann Oncol 2013;24:807-16.

33. Holmes MD, Chen WY, Feskanich D, et al. Physical activity and survival after breast cancer diagnosis. JAMA 2005;293:2479-86.

34. Mishra SI, Scherer RW, Snyder C, et al. Exercise interventions on health-related quality of life for people with cancer during active treatment. Cochrane Database Syst Rev 2012;(8):CD008465.

35. Balduyck B, Sardari Nia P, Cogen A, et al. The effect of smoking cessation on quality of life after lung cancer surgery. Eur J Cardiothorac Surg 2011;40:1432-7; discussion 1437-8.

36. Duffy SA, Terrell JE, Valenstein M, et al. Effect of smoking, alcohol, and depression on the quality of life of head and neck cancer patients. Gen Hosp Psychiatry 2002;24:140-7.

37. Gritz ER, Carmack CL, de Moor C, et al. First year after head and neck cancer: quality of life. J Clin Oncol 1999; 17:352-60.

38. Klosky JL, Tyc VL, Garces-Webb DM, et al. Emerging issues in smoking among adolescent and adult cancer survivors: a comprehensive review. Cancer 2007;110:2408-19.

39. Mariotto AB, Rowland JH, Ries LA, et al. Multiple cancer prevalence: a growing challenge in long-term survivorship. Cancer Epidemiol Biomarkers Prev 2007;16:566-71.

40. Yang G, Wang Y, Wu Y, et al. The road to effective tobacco control in China. Lancet 2015;385:1019-28.

41. Bellizzi KM, Rowland JH, Jeffery DD, et al. Health behaviors of cancer survivors: examining opportunities for cancer control intervention. J Clin Oncol 2005;23:8884-93.

42. Coups EJ, Ostroff JS. A population-based estimate of the prevalence of behavioral risk factors among adult cancer survivors and noncancer controls. Prev Med 2005;40:702-11.

43. Webber K, Davies AN. An observational study to 
determine the prevalence of alcohol use disorders in advanced cancer patients. Palliat Med 2012;26:360-7.

44. Saieva C, Bardazzi G, Masala G, et al. General and cancer mortality in a large cohort of Italian alcoholics. Alcohol Clin Exp Res 2012;36:342-50.

45. Chen W, Xia C, Zheng R, et al. Disparities by province, age, and sex in site-specific cancer burden attributable to 23 potentially modifiable risk factors in China: a comparative risk assessment. Lancet Glob Health

Cite this article as: Li J, Pang H, Sun Z, Zhao L, Bai C. Health status of middle-aged and older cancer survivors: a nationwide cross-sectional study from the China Health and Retirement Longitudinal Study (CHARLS). Ann Transl Med 2020;8(5):183. doi: 10.21037/atm.2020.01.105 2019;7:e257-69.

46. Schmitz KH, Courneya KS, Matthews C, et al. American College of Sports Medicine roundtable on exercise guidelines for cancer survivors. Med Sci Sports Exerc 2010;42:1409-26.

47. Rock CL, Doyle C, Demark-Wahnefried W, et al. Nutrition and physical activity guidelines for cancer survivors. CA Cancer J Clin 2012;62:243-74. 